\title{
Statistics in the landscape of intersecting brane models*
}

\author{
Florian Gmeiner \\ NIKHEF, Kruislaan 409, 1098 SJ Amsterdam, The Netherlands
}

Received: 7 July 2008 / Published online: 23 August 2008

(C) Springer-Verlag / Società Italiana di Fisica 2008

\begin{abstract}
An approach towards a statistical survey of fourdimensional supersymmetric vacua in the string theory landscape is described and illustrated with three examples of ensembles of intersecting D-brane models. The question whether it is conceivable to make predictions based on statistical distributions is discussed. Especially interesting in this context are possible correlations between low energy observables. As an example we look at correlations between properties of the gauge sector of intersecting D-brane models and Gepner model constructions.
\end{abstract}

PACS 11.25.Uv $\cdot$ 11.25.Wx

\section{Introduction}

As has been known for quite a while but being more widely discussed only since recently [1-4], string theory provides us with an extremely large number of effective four-dimensional theories. The main reason for this lies in the abundance of different ways of how to compactify the theory from ten to four dimensions. This procedure is by no means unique and produces massless moduli fields in the effective theory. One way to introduce a potential for these moduli and thereby fix their values is the use of background fluxes (for a recent review in the context of intersecting brane models, see [5]), but even after using this method we are left with an abundance of possibilities.

Facing such a huge number of possible low energy theories, we have to answer the question of why exactly the (supersymmetric) standard model is realised in nature. One certainly has to try to identify a selection mechanism within string theory that singles out a particular solution (e.g. one based on entropy), or one has to face the possibility of retreating to anthropic reasoning.

e-mail: fgmeiner@nikhef.nl

* Based on a talk presented at "The 15th International Conference on Supersymmetry and the Unification of Fundamental Interactions", July 26-August 1, 2007, Karlsruhe, Germany.
However, it might be possible to extract some useful information from a statistical analysis of solutions by searching for common properties within ensembles of models at different points in the landscape. This might give valuable hints for model building by excluding or highlighting regions of the parameter space.

Moreover, looking for correlations between low energy observables might be an interesting possibility [6, 7]. If found, these correlations could not only teach us some lessons about the general behaviour of string theory models and help to identify the underlying principles, but they might even lead to concrete results. If it should turn out that certain correlations exist in a wide variety of models, one could conjecture them to be true in general, thereby obtaining testable predictions for experiment.

Besides these interesting, desired correlations, we have to take a different type of unwanted correlations into account as well [8]. In the analysis of ensembles of models it can be desirable or even necessary to infer from the properties of a particular subset of models the distribution of these features in the whole class. In the simplest case these subsets are randomly chosen, but most of the time one has no other choice but to introduce a bias on the basis of which models are feasible to calculate. In this case one has to be very careful not to run into unwanted correlations. Concretely, the expectation value of the statistical distribution might explicitly depend on the choice function.

How should one proceed to obtain statistical data on string compactifications? There are basically two routes one could follow. One of them relies on a true statistical approach, using an approximated measure for the space of models [9-12]. The other one uses ensembles of explicitly calculated models at specific points in the landscape to compute frequency distributions of their properties, which eventually can be extrapolated to a wider class of compactifications. This is the approach followed here; in particular, we will consider frequency distributions of properties of intersecting brane models [6, 13-20]. Other corners of the landscape that have been studied using a similar method include 
Gepner models [21-24], which we will use later to compare results on correlations, and orbifold compactifications of heterotic string theory [25-27].

\section{Intersecting brane models}

We shall work with simple orientifold models of type IIA, compactified on toroidal orbifold backgrounds and equipped with D6-branes at angles [28]. These models have been studied in great detail over the last years and are still being used for phenomenological model building [29]. However, to be able to study large quantities of different constructions (or even all possible models in a given background), some important points will not be taken into account. One of them is the question of moduli stabilisation, in particular the inclusion of background fluxes, another one concerns the contributions of non-perturbative effects, such as instanton corrections. We believe, however, that the frequency distributions obtained in this simplified setup can be used as a basis for a refined analysis and most of their properties are not going to change qualitatively.

Specifically, the backgrounds we consider are of the form $\mathbb{R}^{1,3} \times M, \quad M=T^{6} / G, G \in\left\{\mathbb{Z}_{2} \times \mathbb{Z}_{2}, \mathbb{Z}_{6}, \mathbb{Z}_{6}^{\prime}\right\}$,

where $\mathbb{Z}_{6}$ and $\mathbb{Z}_{6}^{\prime}$ denote two different embeddings of the group action into the torus-lattice. The orientifold projection consists of dividing out worldsheet parity, accompanied by a reflection along three of the torus axes in space-time. This introduces topological defects, which are described by orientifold O6-planes that carry tension and are charged under the RR-seven-form.

To account for the tadpoles introduced by the O6-planes, one introduces D6-branes in the background, which are space-time filling and wrap, as the orientifold planes, Lagrangian three-cycles in the compact space. These cycles can be parametrised using a basis of $H_{3}(M, \mathbb{Z})$. It can be split into one part that comes from those torus cycles that survive the projections (called bulk cycles in the following) and another part that is due to the existence of exceptional cycles at the fixed points of the orbifold action, which are related to the possible blow-up modes.

For the three geometries under consideration the situation is rather different. In the case of $T^{6} /\left(\mathbb{Z}_{2} \times \mathbb{Z}_{2}\right)$, we do not have exceptional cycles, so that the basis consists of torus cycles only. In the other two cases exceptional cycles do exist and combine with the torus cycles to fractional cycles. The existence of exceptional cycles makes a huge difference for the statistics, as we shall see below.

Not every possible background will lead to a valid model. In order to obtain consistent compactifications, there are several consistency conditions that have to be fulfilled. Two of them have already been alluded to, namely the cancellation of tadpoles coming from the RR-seven-form, which reads in homology

$\sum_{a} N_{a}\left(\pi_{a}+\pi_{a}^{\prime}\right)=L \pi_{\mathrm{O} 6}$,

where the $\pi$ are the three-cycles wrapped by the brane $a$, its orientifold mirror $a^{\prime}$ and the O6-planes. $L$ denotes the orientifold charge, and the sum runs over all stacks of D6branes in the model, each consisting of $N_{a}$ branes. For a background with third Betti number $b_{3}$ there are $b_{3} / 2$ such conditions. Moreover, since we are looking for supersymmetric models in four dimensions, which leads to the constraint that all three-cycles have to be special Lagrangian, we have to impose the condition that the calibration form $\Omega_{3}$ vanishes when restricted to the three-cycle. Additionally anti-branes should be excluded from the spectrum, leading to the condition that the real part of $\Omega_{3}$ has to be positive,

$\left.\operatorname{Im} \Omega_{3}\right|_{\pi}=0, \quad \operatorname{Re} \Omega_{3}>0$.

One last constraint comes from K-theory and can be formulated in our setup as a condition on the intersections between all brane stacks and some orientifold invariant probe-branes,

$\sum_{a} N_{a} \pi_{a} \circ \pi_{\text {probe }}=0 \bmod 2$.

More details on the different geometries, consistency conditions and brane embeddings can be found in $[6,19$, 20].

Each stack of $N$ branes carries a gauge group $G(N)$ on its worldvolume, where $G \in\{U, S p, S O\}$, depending on whether the three-cycle it wraps coincides with the orientifold plane. Matter arises at the intersection of brane stacks and their orientifold mirrors. One can distinguish between chiral and non-chiral multiplets and compute their multiplicities in terms of the intersection numbers between the relevant cycles. For two stacks $a$ and $b$ with $N_{a}$ and $N_{b}$ branes, we obtain chiral matter in the bifundamental representation $\left(\mathbf{N}_{a}, \overline{\mathbf{N}}_{b}\right)$ with multiplicity $I_{a b}=\pi_{a} \circ \pi_{b}$ and in the representation $\left(\mathbf{N}_{a}, \mathbf{N}_{b}\right)$ with multiplicity $I_{a b^{\prime}}=\pi_{a} \circ \pi_{b}^{\prime}$. In a similar manner one can compute the non-chiral multiplets [19]. Moreover, each brane might contribute matter in the adjoint, symmetric and antisymmetric representations of the gauge group $G\left(N_{a}\right)$.

\section{Statistical distributions}

To statistically evaluate the models described in the last section, large ensembles of solutions to the constraints (1), (2) and (3) can be generated using computer algorithms, making use of the fact that after the introduction of a suitable 
basis one can express everything in terms of integer valued algebraic equations.

A complication that arises at this point is that the problem of classifying all possible solutions to the system of equations is NP-complete [30]. However, the number of solutions can be shown to be finite, so it depends on the particular problem of whether it is possible to find all possible solutions within reasonable timescales. In the case of $\mathbb{Z}_{6}^{\prime}$ this is indeed possible, because the set of bulk cycles is very restricted. For the other two backgrounds under consideration an explicit construction of the full space of solutions cannot be achieved, which makes it necessary to impose a restriction to a subset of models. From the properties of the subsets one can then deduce the frequency distributions for the full set of solutions.

The subsets have to be chosen in such a way that no unwanted bias is introduced that would distort the statistical distributions. In the case of $T^{6} /\left(\mathbb{Z}_{2} \times \mathbb{Z}_{2}\right)$ we have used a cut-off on the space of the real part of the complex structure which is one of the free parameters. In this way some interesting models are not captured by the analysis, but the resulting set of solutions is large enough to obtain valid statistical distributions. ${ }^{1}$ For the $T^{6} / \mathbb{Z}_{6}$ background a different method has been used, namely a restriction to several randomly generated subsets of models, that have been tested afterwards to make sure that they do not suffer from unwanted correlations.

The total number of solutions is quite different for the three cases at hand. For $T^{6} /\left(\mathbb{Z}_{2} \times \mathbb{Z}_{2}\right)$ there are $\mathcal{O}\left(10^{10}\right)$ models and in the case of $T^{6} / \mathbb{Z}_{6}$ and $T^{6} / \mathbb{Z}_{6}^{\prime}$ we find $\mathcal{O}\left(10^{28}\right)$ and $\mathcal{O}\left(10^{23}\right)$ solutions, respectively. The huge differences, in particular between the first and the latter two backgrounds, is due to the effect of exceptional branes. One can show that the tadpole constraints (1) split into a bulk part and an exceptional part, so that one can treat the two sets of cycles independently. Each bulk brane can be combined with one of $n_{\mathrm{e}}=2^{7}$ different possible exceptional branes to form a fractional cycle, but not all of them fulfil the consistency conditions, so that the number of possibilities $n_{\mathrm{e}}$ is reduced. For a model with $k$ stacks this amounts to $n_{\mathrm{e}}^{k}$ combinations. Not all of these lead to consistent models, but the restrictions from the exceptional part of the tadpole equations are only polynomial, leading to an exponential enhancement of the space of solutions.

As an example for the frequency distribution of gauge sector properties, we shall take the probability to find a semisimple gauge factor of rank $N$ within one model. Combining the probability of several gauge factors, one can estimate the frequency of certain gauge groups, such as the one

\footnotetext{
${ }^{1}$ If one restricts the survey to models with certain properties, e.g. specific gauge groups, or if one fixes the number of brane stacks, statements about the full set of solutions are possible [18].
}

of the standard model, for example. As one can see clearly from the distributions for the three geometries (Fig. 1), the $T^{6} /\left(\mathbb{Z}_{2} \times \mathbb{Z}_{2}\right)$ orbifold differs quite dramatically from the other two geometries. This is again an effect of the contribution of exceptional cycles, which can be quantified in this case.

For $T^{6} /\left(\mathbb{Z}_{2} \times \mathbb{Z}_{2}\right)$ the distribution for an ensemble of models with a given number of stacks is proportional to $L^{4} / N^{2}$ [18]. Including a sum over all possible stack sizes $k$ and, in the case of models with exceptional stacks, the exponential enhancement factor $n(k)$, all three distributions can be approximated by

$P(N) \approx \sum_{k=1}^{L+1-N} \frac{L^{4}}{N^{2}} n_{\mathrm{e}}^{k}$,

where $n_{\mathrm{e}} \equiv 1$ for models without exceptional cycles. In the case of $T^{6} /\left(\mathbb{Z}_{2} \times \mathbb{Z}_{2}\right)$ we obtain $(L+1) L^{4} / N^{2}-L^{4} / N$, while for the two embeddings of $\mathbb{Z}_{6}$ we find $L^{4} n_{\mathrm{e}}^{(T+1-N)} / N^{2}$.

\section{Correlations}

As mentioned in the introduction, the most promising route to obtain results that may give rise to testable predictions is to look for correlations between low energy observables.

In the following we shall consider only one simple example of correlations between properties of the gauge sector to show how this might be done in practise, but certainly many more possibilities could be considered. ${ }^{2}$

Within the ensemble of models described above, for each pair of branes $a$ and $b$, we always obtain a pair of chiral matter in the bifundamental representation of the two gauge group factors, coming from the intersection of the two branes and the intersection of one brane with the orientifold mirror of the other one. One can define the two quantities

$\Delta^{ \pm}=\left|I_{a b} \pm I_{a b^{\prime}}\right|$

invariant under the exchange of branes and the orientifold action, which describe the net amount of chiral matter for one particular brane.

We shall use these two quantities as an example of how a correlation can arise in the construction, and therefore in the amount of chiral matter that shows up in the effective theory. To show that this effect does not depend on the specific geometry, we compare the analysis of the correlation pattern between intersecting brane models on $T^{6} /\left(\mathbb{Z}_{2} \times \mathbb{Z}_{2}\right)$ and $T^{6} / \mathbb{Z}_{6}$ with a similar analysis of Gepner model constructions [24]. To see how far the actual distribution diverges

\footnotetext{
${ }^{2}$ This section contains some preliminary results of work in progress with Tim Dijkstra.
} 


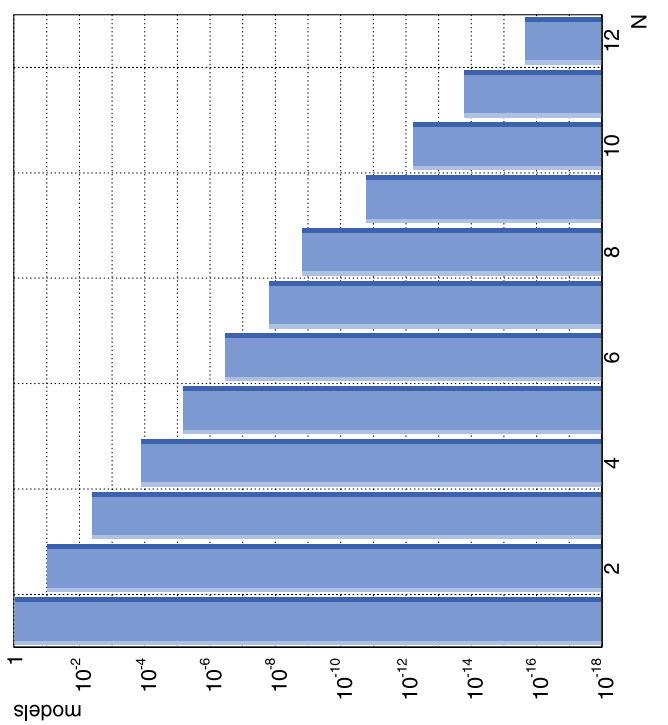

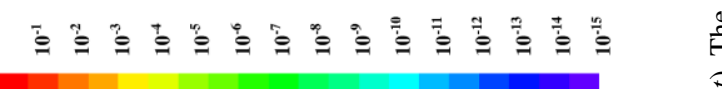
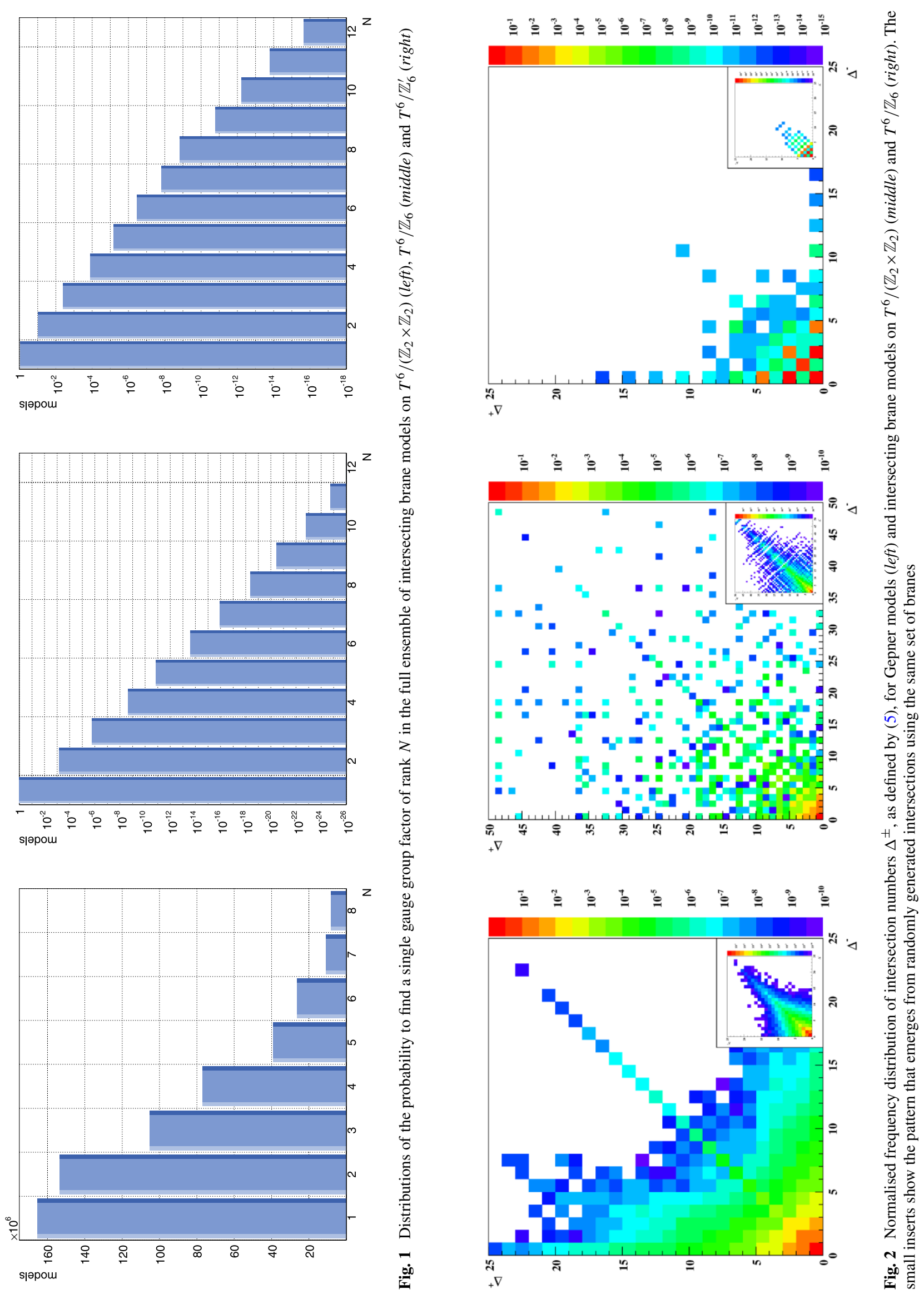
from a generic match of branes, we use a distribution with randomly generated pairings within the set of branes of the model under consideration.

Although the distributions (Fig. 2) are quite different quantitatively, at a qualitative level the distribution of intersection numbers is very similar for the intersecting brane models and the Gepner model constructions. In particular, the tendency towards either identical or rather distant values for $\Delta^{ \pm}$is common in all three distributions. This is quite remarkable, since one has to keep in mind that the Gepner models are not only located at a different point in moduli space, but the ensemble considered here consists of a sample of several thousand different Gepner models, all corresponding to different backgrounds, of which only very few even have a geometrical interpretation.

The fact that the distribution for $T^{6} /\left(\mathbb{Z}_{2} \times \mathbb{Z}_{2}\right)$ looks a bit blurred can be traced back to the fact that the ensemble under consideration has been cut off at high values of the complex structure modulus, as explained above. In the case of $T^{6} / \mathbb{Z}_{6}$, we are considering a random subset of models that include also exceptional branes, which makes the ensemble exponentially larger and at the same time reduces the number of possible values for intersections [19].

Acknowledgment This work is supported by the Dutch Foundation for Fundamental Research of Matter (FOM).

\section{References}

1. W. Lerche, D. Lüst, A.N. Schellekens, Nucl. Phys. B 287, 477 (1987)
2. L. Susskind, arXiv:hep-th/0302219

3. A.N. Schellekens, arXiv:physics/0604134

4. D. Lüst, arXiv:0707.2305[hep-th]

5. R. Blumenhagen, B. Körs, D. Lüst, S. Stieberger, Phys. Rep. 445, 1 (2007)

6. F. Gmeiner, R. Blumenhagen, G. Honecker, D. Lüst, T. Weigand, J. High Energy Phys. 0601, 004 (2006)

7. W. Taylor, Talk in Benasque, July 2007

8. K.R. Dienes, M. Lennek, Phys. Rev. D 75, 026008 (2007)

9. S. Ashok, M.R. Douglas, J. High Energy Phys. 0401, 060 (2004)

10. F. Denef, M.R. Douglas, J. High Energy Phys. 0405, 072 (2004)

11. F. Denef, M.R. Douglas, J. High Energy Phys. 0503, 061 (2005)

12. J. Kumar, Int. J. Mod. Phys. A 21, 3441 (2006)

13. R. Blumenhagen, F. Gmeiner, G. Honecker, D. Lüst, T. Weigand, Nucl. Phys. B 713, 83 (2005)

14. J. Kumar, J.D. Wells, J. High Energy Phys. 0509, 067 (2005)

15. F. Gmeiner, Fortsch. Phys. 54, 391 (2006)

16. F. Gmeiner, M. Stein, Phys. Rev. D 73, 126008 (2006)

17. F. Gmeiner, Fortsch. Phys. 55, 111 (2007)

18. M.R. Douglas, W. Taylor, J. High Energy Phys. 0701, 031 (2007)

19. F. Gmeiner, D. Lüst, M. Stein, J. High Energy Phys. 0705, 018 (2007)

20. F. Gmeiner, G. Honecker, J. High Energy Phys. 0709, 128 (2007)

21. T.P.T. Dijkstra, L.R. Huiszoon, A.N. Schellekens, Phys. Lett. B 609, $408(2005)$

22. T.P.T. Dijkstra, L.R. Huiszoon, A.N. Schellekens, Nucl. Phys. B 710, 3 (2005)

23. P. Anastasopoulos, T.P.T. Dijkstra, E. Kiritsis, A.N. Schellekens, Nucl. Phys. B 759, 83 (2006)

24. T.P.T. Dijkstra, Ph.D. thesis, Nijmegen U. and NIKHEF (2007)

25. K.R. Dienes, Phys. Rev. D 73, 106010 (2006)

26. K.R. Dienes, M. Lennek, D. Senechal, V. Wasnik, Phys. Rev. D 75, 126005 (2007)

27. O. Lebedev et al., Phys. Lett. B 645, 88 (2007)

28. M. Berkooz, M.R. Douglas, R.G. Leigh, Nucl. Phys. B 480, 265 (1996)

29. R. Blumenhagen, M. Cvetič, P. Langacker, G. Shiu, Annu. Rev. Nucl. Part. Sci. 55, 71 (2005)

30. F. Denef, M.R. Douglas, Ann. Phys. 322, 1096 (2007) 